\title{
Acoustically Driven Stark Effect in Transition Metal Dichalcogenide Monolayers
}

\author{
Diego Scolfaro, Matheus Finamor, Luca O. Trinchão, Bárbara L. T. Rosa, Andrey Chaves, \\ Paulo V. Santos, Fernando Iikawa, and Odilon D. D. Couto, Jr.*
}

Cite This: ACS Nano 2021, 15, 15371-15380

ABSTRACT: The Stark effect is one of the most efficient mechanisms to manipulate many-body states in nanostructured systems. In mono- and few-layer transition metal dichalcogenides, it has been successfully induced by optical and electric field means. Here, we tune the optical emission energies and dissociate excitonic states in $\mathrm{MoSe}_{2}$ monolayers employing the $220 \mathrm{MHz}$ in-plane piezoelectric field carried by surface acoustic waves. We transfer the monolayers to high dielectric constant piezoelectric substrates, where the neutral exciton binding energy is reduced, allowing us to efficiently quench (above $90 \%$ )
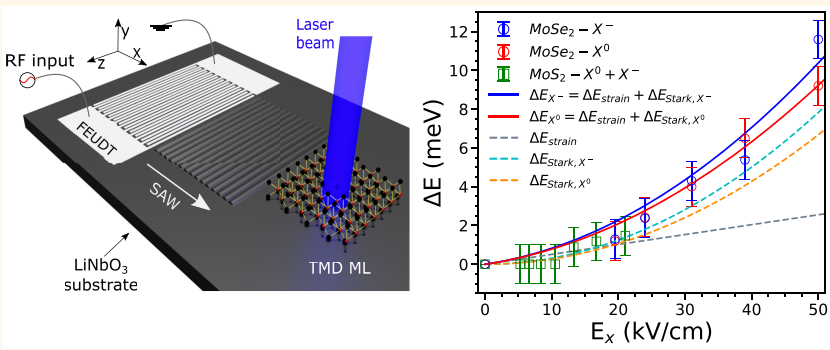

and red-shift the excitonic optical emissions. A model for the acoustically induced Stark effect yields neutral exciton and trion in-plane polarizabilities of 530 and $630 \times 10^{-5} \mathrm{meV} /(\mathrm{kV} / \mathrm{cm})^{2}$, respectively, which are considerably larger than those reported for monolayers encapsulated in hexagonal boron nitride. Large in-plane polarizabilities are an attractive ingredient to manipulate and modulate multiexciton interactions in two-dimensional semiconductor nanostructures for optoelectronic applications.

KEYWORDS: transition metal dichalcogenides, surface acoustic waves, Stark effect, exciton dissociation, dielectric screening, exciton polarizability, trion polarizability

\section{INTRODUCTION}

Transition metal dichalcogenide (TMD) monolayers (MLs) are promising material platforms for future optoelectronic applications due to their multiexciton and spin-valley properties. $^{1-3}$ In order to fulfill the expectations, different approaches have been employed to externally manipulate carriers and the excitonic optical emissions in these systems. ${ }^{4}$ External magnetic fields have been largely used to modify electronic and spin-valley properties. ${ }^{5-8}$ Optical fields ${ }^{9-11}$ and out-ofplane ${ }^{12-15}$ electric fields have been employed to tune emission energies via the Stark effect. In-plane electric fields, besides inducing the Stark effect, can be employed to dissociate excitons and induce lateral carrier transport. ${ }^{16-20}$ Static mechanical strain has also been used to tune emission energies, ${ }^{21,22}$ induce direct-to-indirect band gap transition, ${ }^{23,24}$ change the optical response, ${ }^{25,26}$ and strongly affect carrier dynamics in $2 \mathrm{D}$ systems. ${ }^{27-29}$ The large effective masses and exciton binding energies (compared to III-V semiconductor nanostructures) as well as the strong response to their surroundings are some of the main challenges to achieve ondemand exciton manipulation in TMD MLs. 1,30

The integratability of surface acoustic wave (SAW) devices with planar systems holds promising perspectives for applications in photonics, optomechanics, and quantum information science. ${ }^{31,32}$ The propagating mechanical deformation (strain) and piezoelectric potential of SAWs have been successfully employed to transport carrier and spin packets in semiconductor quantum wells $s^{33-35}$ and to modulate the optical emission of single nanostructures. ${ }^{36-40}$ The employment of SAWs to manipulate the optical properties of few-layer TMDs, however, is a field in its infancy. ${ }^{41,42}$ In MLs, the few so far reported experiments have been performed exclusively at room temperature on $\mathrm{MoS}_{2}$ deposited on non-piezoelectric surfaces, ${ }^{43,44}$ thus relying solely on the ML piezoelectricity ${ }^{45}$ induced by the SAW mechanical deformation to change the carrier dynamics. A photoluminescence (PL) quenching followed by an energy blue-shift of the neutral exciton + trion $\left(X^{0}+X^{-}\right)$emission band of $\mathrm{MoS}_{2}$ with increasing acoustic power has been observed. The blue-shift of the broad $X^{0}+X^{-}$emission band toward the neutral exciton $\left(X^{0}\right)$

Received: August 9, 2021

Accepted: August 25, 2021

Published: August 27, 2021 


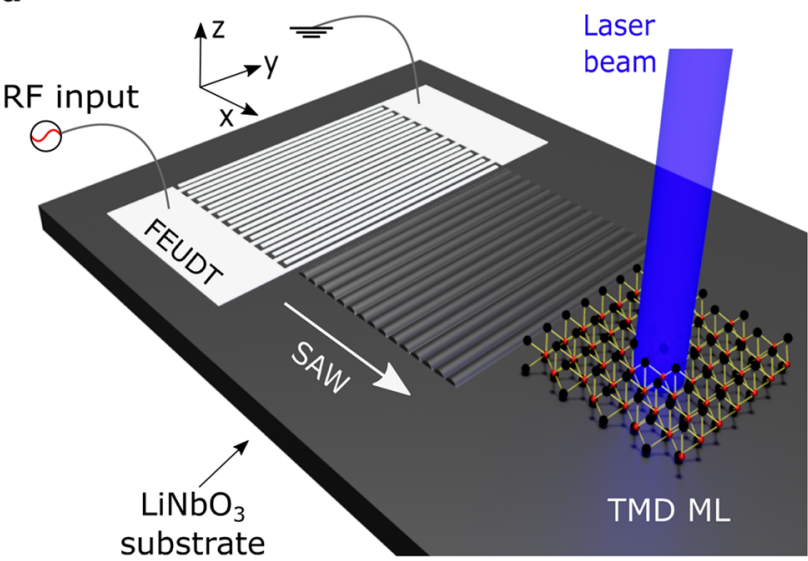

b

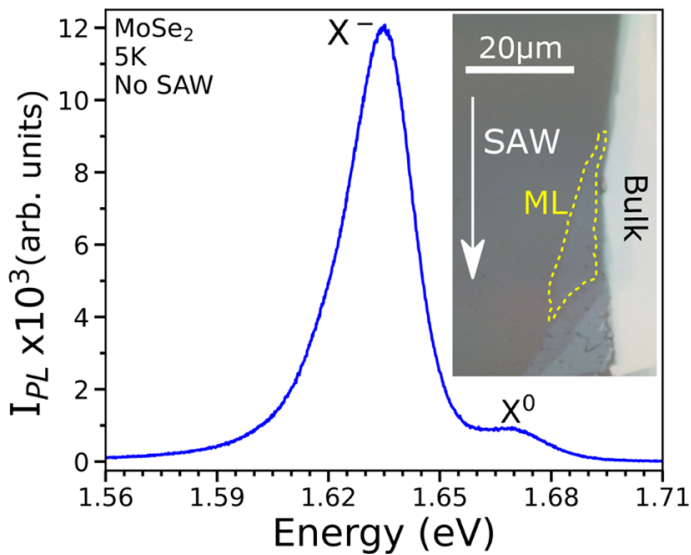

Figure 1. Acoustic modulation experiment. (a) Device illustration: the SAW beam is launched along the $x$ direction by a FEUDT device toward the TMD ML, where PL is excited by a focused laser beam and collected by an optical objective (not shown). (b) Inset: optical micrograph of the mechanically exfoliated $\mathrm{MoSe}_{2}$ flake on the $\mathrm{LiNbO}_{3}$ substrate. The $\mathrm{ML}$ is bounded by the dashed line region at the side of the thicker region of the flake (bulk). The vertical arrow indicates the SAW propagation direction. Main plot: low-temperature (5 K) $\mu$-PL spectrum of the $\mathrm{MoSe}_{2}$ ML without the SAW. The $X^{0}$ and $X^{-}$emission lines are indicated.

a

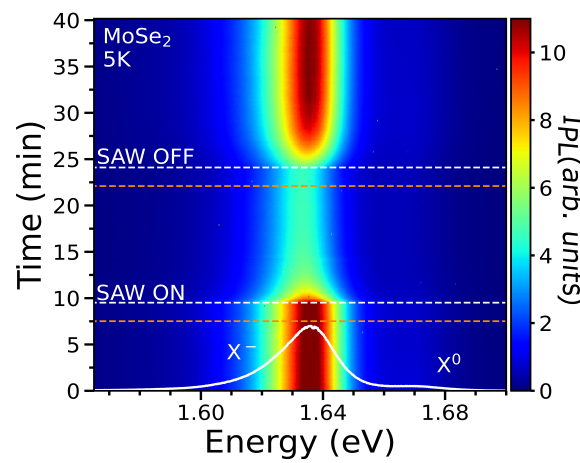

d

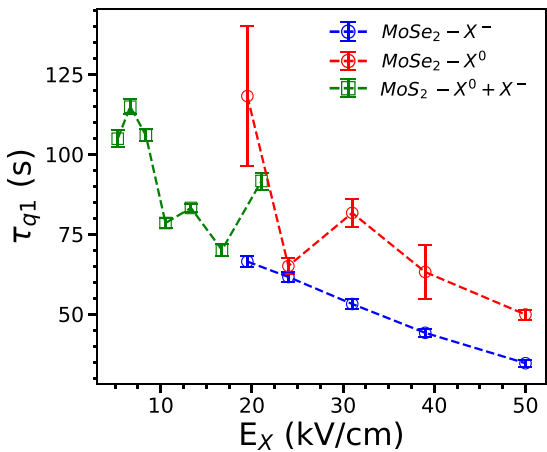

b

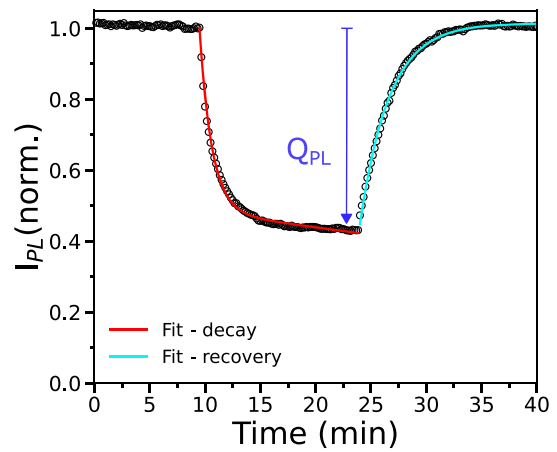

e

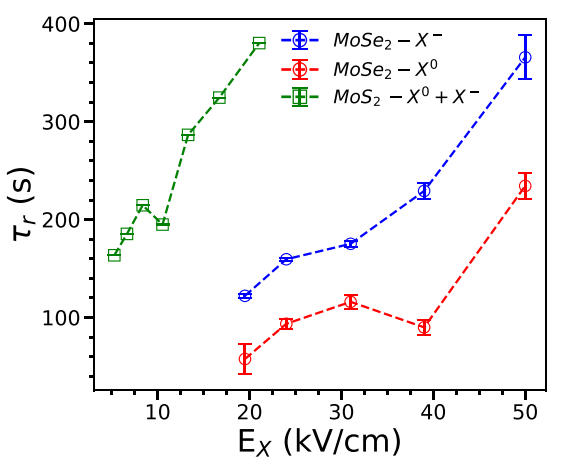

C

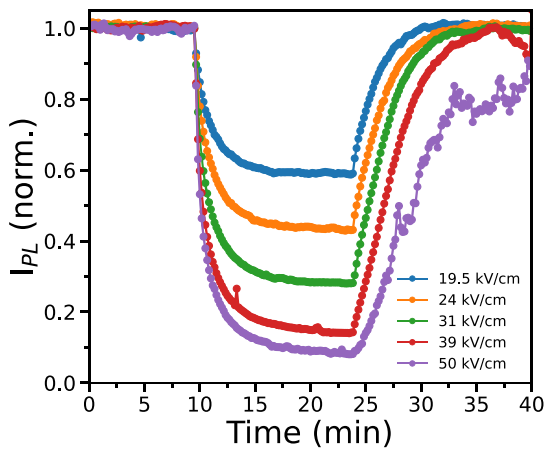

$\mathbf{f}$

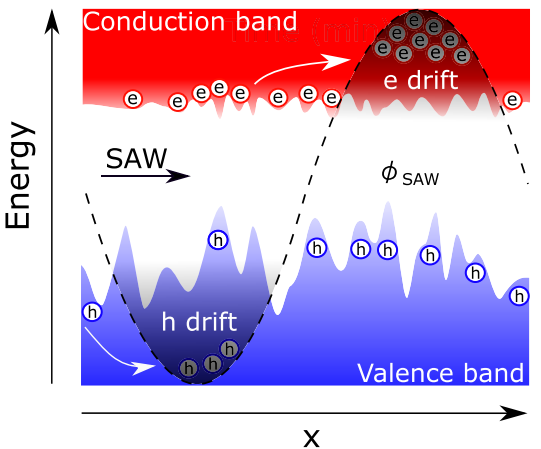

Figure 2. SAW effect on the low-temperature emission of TMD MLs. (a) Map of the MoSe $\mathrm{e}_{2}$ ML PL emission as a function of time recorded at $5 \mathrm{~K}$. The white horizontal dashed lines indicate when the $\mathrm{SAW}\left(E_{x}=24 \mathrm{kV} / \mathrm{cm}\right)$ is turned on and off during the experiment. The horizontal orange lines indicate the time window of $2 \mathrm{~min}$ from where PL is averaged to calculate the degree of PL quenching ( $\left.Q_{\mathrm{PL}}\right)$. The white spectrum illustrates the PL emission before the SAW is turned on. (b) Fits of the PL decay (red solid line) and recovery (cyan solid line) transients of the $X^{-}$emission extracted from (a). (c) Transients of the $\mathrm{X}^{-}$PL emission of the $\mathrm{MoSe}_{2}$ ML for different values of $E_{x}$. (d) PL quenching $\left(\tau_{\mathrm{q} 1}\right)$ and (e) recovery $\left(\tau_{\mathrm{r}}\right)$ times as a function of $E_{x}$ for the $\mathrm{X}^{-}$(blue circles) and $X^{0}$ (red circles) emission lines of the MoSe $\mathrm{ML}_{2}$ and the $X^{0}+X^{-}$emission band of the $\mathrm{MoS}_{2} \mathrm{ML}$ (green squares). The error bars represent the fitting uncertainties. (f) Illustration of the conduction and valence bands' potential fluctuations along the carrier transport path. The SAW-modulated piezoelectric potential $\phi_{\mathrm{SAW}}($ not to scale with the potential fluctuations) is represented by the dashed line.

transition has been attributed to a more efficient trion $\left(X^{-}\right) \mathrm{PL}$ quenching as compared to $X^{0}$ due to the 1 order of magnitude larger binding energy of the latter.

The large $X^{0}$ binding energies in TMD MLs on substrates with low dielectric constants make these excitonic complexes relatively insensitive to the SAW fields. Here, we demonstrate the efficient acoustic control of excitonic resonances in $\mathrm{MoSe}_{2}$ MLs placed on high dielectric constant $\mathrm{LiNbO}_{3}$, where the $X^{0}$ binding energy is strongly reduced, thus becoming significantly smaller than in MLs encapsulated in hexagonal boron nitride 
or on $\mathrm{SiO}_{2} / \mathrm{Si}$ substrates. ${ }^{1,2,8}$ We show that a $220 \mathrm{MHz}$ dynamic SAW field can efficiently tune the energy and strength of the excitonic optical transitions. The spectrally separated $X^{0}$ and $X^{-}$emission lines of $\mathrm{MoSe}_{2}$ MLs red-shift quadratically with the amplitude of the SAW longitudinal piezoelectric field. We present a model for the acoustically induced Stark effect which yields large in-plane polarizabilities of $\alpha_{\mathrm{X}}{ }^{0}=(530 \pm 50)$ $\times 10^{-5} \mathrm{meV} /(\mathrm{kV} / \mathrm{cm})^{2}$ and $\alpha_{\mathrm{X}^{-}}=(630 \pm 70) \times 10^{-5} \mathrm{meV} /$ $(\mathrm{kV} / \mathrm{cm})^{2}$ for $X^{0}$ and $X^{-}$, respectively. The slightly larger $X^{-}$inplane polarizability is consistent with the fact that $X^{-}$and $X^{0}$ binding energies are no longer 1 order of magnitude different. Our results also indicate that, under strong dielectric screening, the excitonic interaction in TMD MLs has to be described beyond the Rytova-Keldysh potential by taking into account the whole dielectric constant dispersion of the system. ${ }^{46}$ Furthermore, the lower binding energies enable efficient multiexciton dissociation and carrier transport by the moving SAW fields. The PL quenching transients indicate that electrons are drifted more efficiently by the acoustic fields than holes. This shows that the large potential fluctuations due to $\mathrm{ML} /$ substrate coupling are another major factor limiting long-range coherent ambipolar carrier transport by the SAWs in TMD MLs. These potential fluctuations are more pronounced in the valence band (compared to the conduction band) and limit hole mobility. ${ }^{47,48}$ Similar results are obtained in $\mathrm{MoS}_{2}$ MLs. Our achievements prove that the SAW piezoelectric fields can be employed as a high-frequency reliable tool to modulate many-body states in TMD MLs and create interesting perspectives for heterostructure engineering $^{49}$ for coherent carrier packet transport in $2 \mathrm{D}$ semiconductor systems.

\section{RESULTS AND DISCUSSION}

Figure 1a illustrates the experimental configuration. The MLs were placed in front of floating electrode unidirectional transducers (FEUDTs) ${ }^{50}$ which, under radio frequency excitation at the SAW resonance frequency, launch propagating acoustic beams along the $\mathrm{X}$ direction of $\mathrm{Y}-128^{\circ} \quad \mathrm{LiNbO}_{3}$ piezoelectric substrates ( $x$ direction in our frame of reference). The SAW induces a modulated mechanical deformation (strain) and a piezoelectric potential that propagate with a well-defined velocity $\left(v_{x}\right)$ and interact with the excitonic complexes excited in the MLs at low temperatures. In the experiments, the optical excitation is performed with a focused laser beam, and the $\mu$-PL emission is monitored initially without the SAW. After some minutes, the SAW is turned on and the emission of the MLs is monitored as a function of time. The inset of Figure $1 \mathrm{~b}$ shows a microscope image of the $\mathrm{MoSe}_{2}$ flake transferred to the $\mathrm{LiNbO}_{3}$ surface. The dashed line region marks the boundaries of the ML. The SAW propagation direction is indicated by the white arrow. The main plot in Figure $1 \mathrm{~b}$ shows the $5 \mathrm{~K} \mu$-PL spectrum of the $\mathrm{MoSe}_{2} \mathrm{ML}$ in the absence of the SAW. The two main PL emission lines at 1.635 and $1.670 \mathrm{eV}$ are attributed to the $X^{-}$ and $X^{0}$ excitonic complexes, respectively. The approximately $15 \mathrm{meV}$ blue-shift of the emission lines in comparison to samples on $\mathrm{SiO}_{2} / \mathrm{Si}$ substrates is attributed to the net effect of the band gap renormalization and the strong decrease of the $X^{0}$ binding energy in high dielectric constant $\mathrm{LiNbO}_{3}$ substrates. ${ }^{17,30}$ The energy splitting between the $X_{0}$ and the $X^{-}$ emissions will be assumed to be the $X^{-}$binding energy $E_{\mathrm{B}}^{X-}=$ $35 \mathrm{meV}$ (for further discussion see the Supporting Information). This value is similar to the ones reported for
$\mathrm{MoSe}_{2} \mathrm{MLs}$ on different substrate systems. It is in agreement with recent results that indicate that, in contrast to the $X^{0}$, the $X^{-}$binding energy is more dependent on the TMD ML constituents (transition metal and chalcogen atomic sheets) than on the substrate underneath. ${ }^{51}$

Figure $2 \mathrm{a}$ shows a $\mu$-PL spectral map assembled by sequentially recording $\mathrm{MoSe}_{2} \mathrm{ML}$ spectra as a function of time starting with the SAW turned off. Each spectrum was recorded with an integration time of $10 \mathrm{~s}$. The emission was monitored by approximately $10 \mathrm{~min}$ (indicated by the dashed horizontal white line), when a SAW with an in-plane piezoelectric field $E_{x}=24 \mathrm{kV} / \mathrm{cm}$ was turned on (see the Methods section and the Supporting Information for details about the power to electric field conversion as well as about the finite element simulations of the FEUDT devices where the $\mathrm{MoSe}_{2}$ and $\mathrm{MoS}_{2}$ MLs have been exfoliated). Under the SAW, the emission immediately changes and the intensity slowly decreases. When the SAW is turned off (after approximately 15 $\mathrm{min}$ ), the emission recovers its initial levels. By integrating (in energy) the PL intensity around each emission line, we can discriminate the effect of the SAW on the $X^{0}$ and $X^{-}$emissions. Figure $2 \mathrm{~b}$ presents the integrated (and background subtracted) time transient of the $X^{-}$emission shown in Figure 2a. The profile is normalized by the PL intensity in the absence of the SAW. In this way, as indicated by the blue arrow, we can quantify the degree of PL quenching as $Q_{\mathrm{PL}}=1-I_{\mathrm{PL}}\left(t_{\mathrm{OFF}}\right)$, where $I_{\mathrm{PL}}\left(t_{\mathrm{OFF}}\right)$ is the normalized PL intensity when the SAW is turned off at the time $t=t_{\mathrm{OFF}}$.

The PL quenching induced by a SAW has been demonstrated to be an important measure of the ambipolar carrier transport efficiency in high-quality III-V semiconductor quantum wells. ${ }^{33-35,52}$ In such experiments, the modulated SAW piezoelectric potential induces a type-II spatial carrier separation along the SAW wavelength, which ionizes optically excited excitons and transports the resulting electrons (holes) in the maxima (minima) of the potential with the SAW velocity. In this way, the acoustically induced PL quenching arises from (i) exciton ionization followed by (ii) spatial separation of the free electrons and holes and by (iii) acoustic transport out of the detection area. In addition, the SAW can increase the radiative recombination lifetime and, thus, may favor nonradiative recombination channels, which also cause some PL quenching. The exciton ionization rate depends on the piezoelectric field amplitude and on excitonic properties such as binding energy and effective mass. ${ }^{53}$

The PL quenching transients in our experiments could only be fitted with two exponential decay constants, namely, $\tau_{\mathrm{q} 1}$ and $\tau_{\mathrm{q} 2}$. The result is represented by the red solid line in Figure $2 \mathrm{~b}$, where $\tau_{\mathrm{q} 1}=60 \mathrm{~s}$ and $\tau_{\mathrm{q} 2} \approx 100 \mathrm{~min}$. These values are consistent with photocurrent measurements reported in (inplane) field effect devices fabricated with TMD MLs ${ }^{16-18,20}$ and with photoconductivity measurements using SAWs in IIVI quantum wells. ${ }^{54}$ The slow decrease (buildup) of the photocurrent in the presence (absence) of an in-plane field has been attributed to persistent photoconductivity (PPC) associated with potential fluctuations along the carrier transport path. Recent experiments indicate that, for nonencapsulated MLs, the local valence band potential fluctuations associated with strain due to coupling with the substrate are larger than the conduction band ones, making electrons more mobile than holes on the TMD plane ${ }^{47,48}$ ( $c f$. Figure 2f). In field effect devices, decay constants between $10 \mathrm{~s}$ and $2 \mathrm{~min}$ (consistent with $\tau_{q 1}$ ) have been attributed to electron trapping 
a

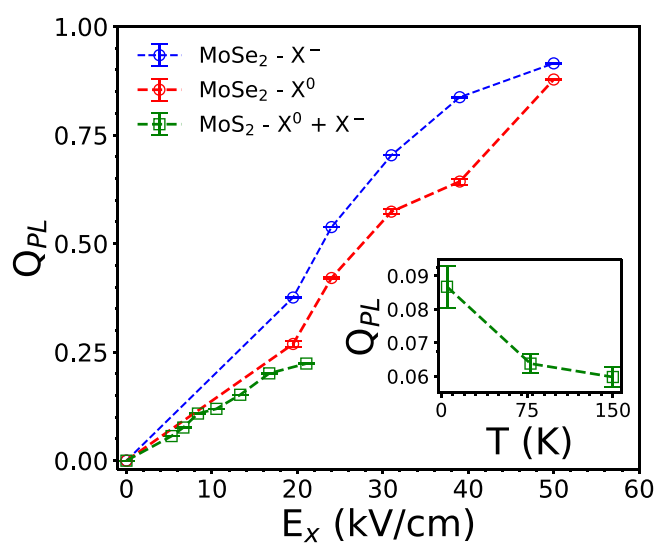

b

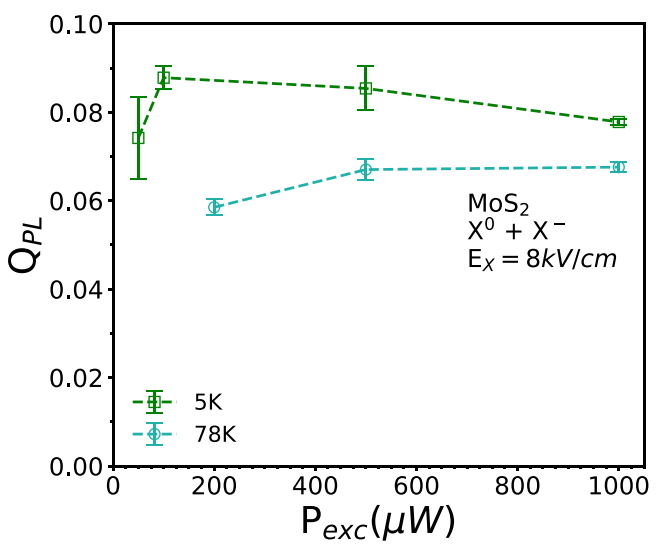

Figure 3. Piezoelectric field, temperature, and laser excitation power dependence of the degree of PL quenching $\left(Q_{P_{L}}\right)$. (a) $Q_{P L}$ as a function of $E_{x}$ for the $X^{-}$(blue circles) and $X^{0}$ (red circles) emission lines of the $\mathrm{MoSe}_{2} \mathrm{ML}$ and the $X^{0}+X^{-}$emission (green squares) of the MoS $\mathrm{ML}_{2}$ Inset: temperature dependence of $Q_{\mathrm{PL}}$ for the $\mathrm{MoS}_{2}$ emission at $E_{x}=8 \mathrm{kV} / \mathrm{cm}$. (b) $Q_{\mathrm{PL}}$ as a function $P_{\text {exc }}$ at $5 \mathrm{~K}$ (green squares) and $78 \mathrm{~K}$ (light green circles) for the $\mathrm{MoS}_{2} \mathrm{ML}$ emission band measured at $8 \mathrm{kV} / \mathrm{cm}$. The error bars represent the uncertainties due to PL intensity averaging.

and release processes at these potential fluctuations during transport induced by the in-plane electric fields. ${ }^{16,48}$

Figure $2 \mathrm{c}$ presents the PL quenching transients for the $X^{-}$ emission of the $\mathrm{MoSe}_{2}$ ML for different amplitudes of the inplane SAW piezoelectric field, demonstrating that the degree of PL quenching $Q_{\mathrm{PL}}$ clearly increases with $E_{x}$. The same behavior is observed for the $X^{0}$ emission line as well as for the broad $\left(X^{0}\right.$ $+X^{-}$) emission of the $\mathrm{MoS}_{2} \mathrm{ML}$ (see the Supporting Information). Figure $2 \mathrm{~d}$ shows the dependence of $\tau_{\mathrm{q} 1}$ on $E_{x}$, summarizing the results obtained for the $\mathrm{MoSe}_{2}$ and $\mathrm{MoS}_{2}$ MLs. There is a clear decrease in $\tau_{\mathrm{q} 1}$ as $E_{x}$ increases. Also, the $\mathrm{MoSe}_{2}$ and $\mathrm{MoS}_{2}$ MLs behave in a similar way if SAW fields of the same magnitude are generated in each sample (which is expected, since $\mathrm{MoSe}_{2}$ and $\mathrm{MoS}_{2}$ have similar material parameters). Finally, for the $\mathrm{MoSe}_{2} \mathrm{ML}$, the decay of the $X^{0}$ emission seems to be consistently slower than the one of $X^{-}$.

The 15 min time window under acoustic power that we probed in our experiments does not allow us to precisely determine the whole dependence of the long decay time constant $\tau_{\mathrm{q} 2}$ on $E_{x}$. However, our double exponential decay fit indicates that $\tau_{\mathrm{q} 2}$ also decreases with $E_{x}$. For the highest value of $E_{x}(50 \mathrm{kV} / \mathrm{cm})$ we obtain $\tau_{\mathrm{q} 2} \approx 12$ min for the $X^{-}$emission line of the $\mathrm{MoSe}_{2} \mathrm{ML}$, which is already comparable to our experimental time window and considerably smaller than the value of $\tau_{\mathrm{q} 2} \approx 100 \mathrm{~min}$ obtained for $E_{x}=24 \mathrm{kV} / \mathrm{cm}$. Since we do not have electrical contacts on the MLs (which could lead to other transient effects ${ }^{16}$ ), $\tau_{\mathrm{q} 2}$ can possibly be related to hole drift by the in-plane SAW field. In this way, our results suggest that electrons are drifted away from the laser generation spot (at rates associated with $\tau_{\mathrm{q} 1}$ ) more efficiently than holes (at rates associated with $\tau_{\mathrm{q} 2}$ ). This is a consequence of the uneven balance of conduction and valence band potential fluctuations along the carrier transport path, ${ }^{16,48}$ as depicted in Figure $2 f$. The transport rates for both carriers, however, increase as the in-plane SAW piezoelectric field amplitude is increased, thus indicating that higher acoustic fields or larger mobilities could lead to more efficient acoustic carrier transport in $2 \mathrm{D}$ systems.

The cyan solid line in Figure $2 \mathrm{~b}$ is a fit of the experimental data with a buildup transient $I_{\mathrm{PL}}(t)=1-Q_{\mathrm{PL}} \exp [-(t-$ $\left.t_{\mathrm{OFF}}\right) / \tau_{\mathrm{r}}$, where $\tau_{\mathrm{r}}$ is the PL recovery time. ${ }^{55}$ Figure $2 \mathrm{e}$ shows that the recovery process is slower than the quenching induced by the high-frequency SAW. It also shows that for both MLs, as $E_{x}$ increases, more time is needed to recover the initial PL intensity. The PL recovery process at the laser generation spot after the electron and hole populations are drifted by the SAW possibly follows a complex dynamics which may depend of the rearrangement of carriers in the (shallow and deep) potential fluctuations in/around each ML (as reported in field effect devices ${ }^{18,20,48}$ ), carrier tunneling from the substrate, or even rearrangement of adatoms on the ML surface after the in-plane piezoelectric field is turned off. This explains why, differently from the quenching process, which is determined only by the amplitude of the driving SAW piezoelectric field, the recovery process without the SAW seems to be more characteristic of each sample.

Figure 3a summarizes the dependence of the degree of PL quenching $Q_{\mathrm{PL}}$ on $E_{x}$ for the $\mathrm{MoSe}_{2}$ and $\mathrm{MoS}_{2}$ MLs. $Q_{\mathrm{PL}}$ increases approximately linearly with the amplitude of the inplane SAW piezoelectric field. At high acoustic powers, the $\mathrm{MoSe}_{2} X^{-}$emission line is quenched by more than $90 \%$, thus indicating an effective SAW-induced exciton dissociation and carrier drift process. The inset in Figure 3a displays the temperature dependence of the $\mathrm{MoS}_{2} X^{0}+X^{-}$emission band measured at a very low acoustic power (corresponding to $E_{x}=$ $8 \mathrm{kV} / \mathrm{cm}$ ). It shows that the PL quenching degree decreases when the temperature increases (possibly due to the extra thermal energy, which makes the carrier drift process less efficient). The latter indicates no significant contribution of SAW-induced heating effects to the results presented in the main plot of Figure $3 \mathrm{a}$, where $Q_{\mathrm{PL}}$ steadily increases with the acoustic power (for further discussion about SAW-induced heating see the Supporting Information). Figure $3 \mathrm{~b}$ presents the behavior of $Q_{\mathrm{PL}}$ for the excitonic emissions of $\mathrm{MoS}_{2}$ as a function of laser excitation power $\left(P_{\text {exc }}\right)$ measured at two different temperatures $\left(5\right.$ and $78 \mathrm{~K}$ ) again with $E_{x}=8 \mathrm{kV} / \mathrm{cm}$. No drastic change or trend in $Q_{\mathrm{PL}}$ can be detected in the range of excitation powers used in the experiments. This means that even at relatively weak acoustic fields (i) the PL quenching efficiency does not decrease with the excited exciton density up to liquid nitrogen temperatures and (ii) no laser-induced heating can be detected in the experiments. 
a

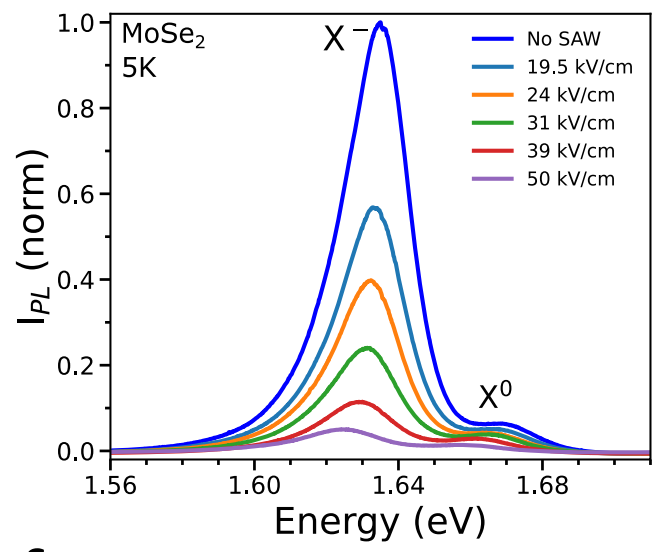

C

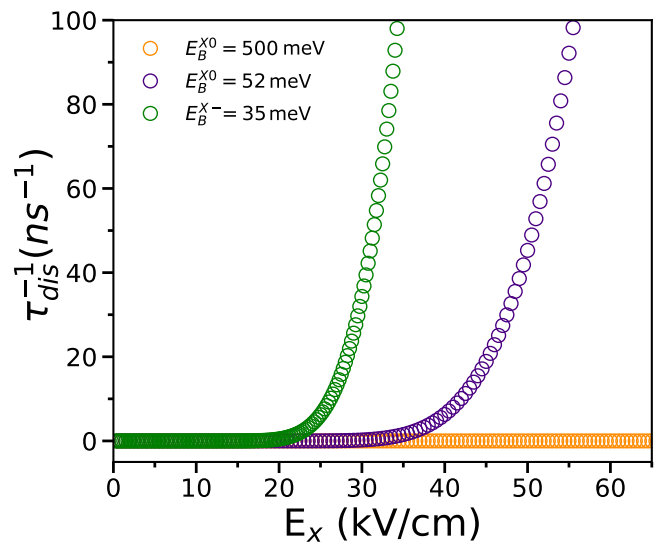

b

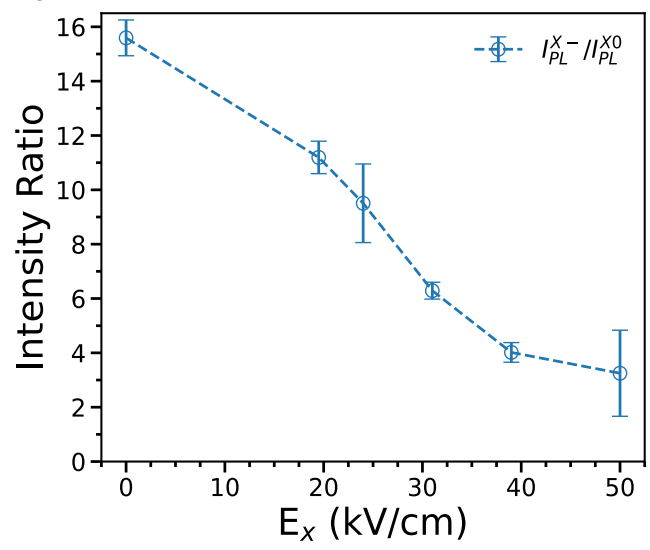

d

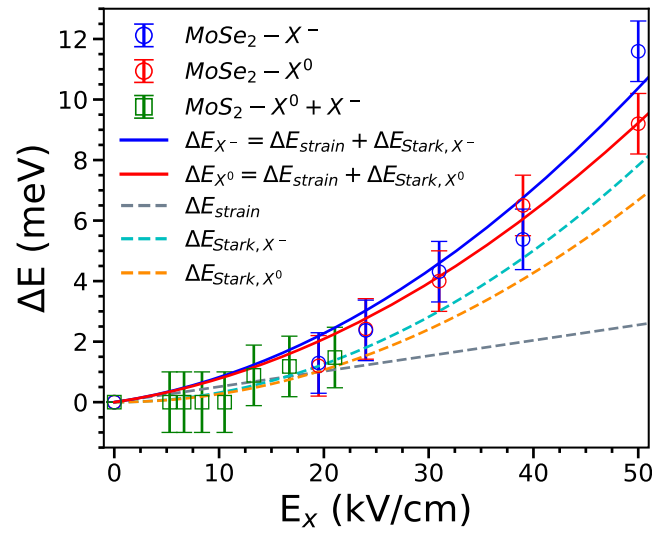

Figure 4. Acoustically induced Stark effect. (a) $\mathrm{MoSe}_{2}$ ML PL spectra (average for 2 min) before the SAW is turned on (blue spectrum) and for different in-plane SAW fields (remaining spectra) before it is turned off. (b) $X^{-}$and $X^{0}$ intensity ratio $I_{\mathrm{PL}}^{X^{-}} / I_{\mathrm{PL}}^{X^{0}}$ as a function of $E_{x}$ extracted from the spectra shown in (a). (c) Calculated exciton dissociation rates as a function of $E_{x}$ for $E_{\mathrm{B}}^{X^{0}}=500 \mathrm{meV}$ (orange dots) and $52 \mathrm{meV}$ (purple dots) as well as for $E_{\mathrm{B}}^{X^{-}}=35 \mathrm{meV}$ (green dots). (d) $\mathrm{MoSe}_{2} \mathrm{ML} \mathrm{X}^{-}$(blue circles) and $X^{0}$ (red circles) emissions red-shift ( $\Delta E$ ) as a function of $E_{x}$. The gray dashed line is the calculated hydrostatic strain contribution $\left(\Delta E_{\text {strain }}\right)$. The solid blue and red lines are fits to the experimental data of the $X^{-}$and $X^{0}$ emissions with eq 2, respectively. The dashed cyan and orange lines are the Stark shift contributions $\left(\Delta E_{\text {Stark }}\right)$ to $\Delta E$ extracted from fits. The values of $\Delta E$ for the $X^{0}+X^{-}$emission of the $\mathrm{MoS}_{2} \mathrm{ML}$ (green squares) are also shown.

Figure $4 \mathrm{a}$ presents the behavior of the $X^{0}$ and $X^{-} \mu$-PL spectra of the $\mathrm{MoSe}_{2} \mathrm{ML}$ with $E_{x}$. As a reference, the blue solid line is the ML spectrum in the absence of the SAW (averaged for 2 min before the SAW is turned on). The remaining spectra for different values of $E_{x}$ are averages of the spectra recorded for $2 \mathrm{~min}$ before the SAW is turned off. We observe that the $X^{-}$ emission is more affected by the acoustic fields than the $X^{0}$ one. Figure $4 \mathrm{~b}$ shows the ratio $I_{\mathrm{PL}}^{X-} / I_{\mathrm{PL}}^{\mathrm{X} 0}$ between the two integrated intensities as a function of $E_{x}$. The ratio drops from a factor of more than 15 times in the absence of the SAW to about 3 times at high acoustic fields, demonstrating that the SAW quenches more efficiently the trion emission as compared to the neutral exciton one (for the behavior of $I_{\mathrm{PL}}^{X-} / I_{\mathrm{PL}}^{X 0}$ with $P_{\text {exc }}$ see the Supporting Information). This is consistent with the larger $Q_{\mathrm{PL}}$ (shown in Figure 3a) for the $X^{-}$emission in comparison to the $X^{0}$ one and with the fact that the $X^{0}$ binding energy is larger than the $X^{-}$one (see discussion below).

Approximating the ML by a thin quantum well, we can estimate the SAW-induced exciton dissociation rate $\left(\tau_{\text {dis }}^{-1}\right)$ in terms of the in-plane piezoelectric field $\left(E_{x}\right)$ and exciton binding energy $\left(E_{\mathrm{B}}\right):^{42,53}$

$$
\frac{1}{\tau_{\mathrm{dis}}}=\frac{8 E_{\mathrm{B}}}{\hbar \sqrt{\pi}}\left(\frac{E_{\mathrm{B}}}{2 e \hbar E_{x}} \sqrt{2 \mu E_{\mathrm{B}}}\right)^{1 / 2} \exp \left(-\frac{4 E_{\mathrm{B}}}{3 e \hbar E_{x}} \sqrt{2 \mu E_{\mathrm{B}}}\right)
$$

where $E_{\mathrm{B}}=E_{\mathrm{B}}^{X^{0}}$ for $X^{0}$ or $E_{\mathrm{B}}=E_{\mathrm{B}}^{X^{-}}=35 \mathrm{meV}$ for $X^{-}$. We consider the electron and hole effective masses as $0.49 m_{0}$ and $0.58 m_{0}^{56}$ ( $m_{0}$ is the free electron mass), respectively. In this way, we obtain $\mu=\mu_{X^{0}}=0.266 m_{0}$ and $\mu=\mu_{X^{-}}=0.364 m_{0}$ for the reduced masses for $X^{0}$ and $X^{-}$complexes in the $\mathrm{MoSe}_{2} \mathrm{ML}$, respectively. The value of $E_{\mathrm{B}}^{X^{0}}=52 \mathrm{meV}$ was calculated within the effective mass model considering an effective dielectric constant of $56 \varepsilon_{0}$ for the $\mathrm{Y}-128^{\circ} \mathrm{LiNbO}_{3}$ substrate $^{57}\left(\varepsilon_{0}\right.$ is the vacuum permittivity). In order to be consistent with the experimental results and account for such strong dielectric screening, the electron-hole interaction in our system could only be satisfactorily described by considering the full dispersion of the substrate dielectric constant, ${ }^{46}$ thus showing the limitation of the Rytova-Keldysh approximation ${ }^{58,59}$ in our case (see the Supporting Information for the discussion about the exciton modeling).

Figure $4 \mathrm{c}$ shows the exciton dissociation rates calculated with eq 1 as a function of $E_{x}$. For $E_{\mathrm{B}}^{X^{-}}=35 \mathrm{meV}$, the $X^{-}$ 
dissociation rate for $\mathrm{MoSe}_{2} \mathrm{MLs}$ on $\mathrm{LiNbO}_{3}$ increases rapidly for fields above $20 \mathrm{kV} / \mathrm{cm}$. In the experiments, the onset of PL quenching is observed at even lower values. For $\mathrm{MoSe}_{2}$, at the lowest measured acoustic power (corresponding to $E_{x}=19.5$ $\mathrm{kV} / \mathrm{cm}) Q_{\mathrm{PL}}$ was approximately $30 \%$. For $\mathrm{MoS}_{2}$, in-plane fields on the order of $10 \mathrm{kV} / \mathrm{cm}$ already yielded some PL quenching. The predictions obtained with eq 1 , which are displayed in Figure $4 c$, are, nevertheless, in very good agreement with the experiments in the sense of showing that the onset of PL quenching associated with the $X^{-}$dissociation (which is the first step for an efficient carrier transport) is expected at fields that are very similar to the experimental values. The SAW modulated strain may also have a contribution to exciton dissociation, ${ }^{24,60}$ as discussed below, explaining the onset at fields lower than $20 \mathrm{kV} / \mathrm{cm}$ observed in the experiments. Note also that, as long as the expected dissociation rates associated with the piezoelectric field amplitude increase above $10 \mathrm{~ns}^{-1}$, they become of the same order of magnitude of the expected $X^{-}$recombination rates, ${ }^{61}$ thus showing that the $\mathrm{PL}$ quenching appears due to the competition between the dissociation induced by the SAW and the radiative recombination.

The effect of the dielectric screening is also analyzed in Figure $4 \mathrm{c}$. We consider $E_{\mathrm{B}}^{X^{0}}=500 \mathrm{meV}$ and $E_{\mathrm{B}}^{X^{0}}=52 \mathrm{meV}$ to compare situations where the effective dielectric constant is low and high, respectively. For substrates with a low effective dielectric constant, in-plane fields like the ones achieved in our devices would lead to negligible dissociation rates due to the large $X^{0}$ binding energy. However, for $E_{\mathrm{B}}^{X^{0}}=52 \mathrm{meV}$ the onset of the neutral exciton dissociation is expected to happen at inplane fields of the same order of magnitude as the ones employed in our experiments. The larger in-plane field expected for the onset of the $X^{0} \mathrm{PL}$ quenching as compared to the $X^{-}$one is consistent with the larger $X^{0}$ binding energy. This is in full agreement with the result shown in Figure $4 b$, where we see a faster decrease of $I_{\mathrm{PL}}^{X^{-}}$as compared to $I_{\mathrm{PL}}^{X^{0}}$ as $E_{x}$ is increased. In the experiments, we also observe that $I_{\mathrm{PL}}^{X^{0}}$ starts to quench for fields lower than the expected $35 \mathrm{kV} / \mathrm{cm}$. This can also be due to the SAW strain. Another important contribution may come from impact ionization due to electrons accelerated by the large piezoelectric fields, which has led to much lower quenching onsets than expected for the $X^{0}$ emission in GaAs quantum wells. $^{62}$ Moreover, as long as the $X^{-}$dissociation starts, the $X^{0}$ dissociation rate may increase due to the increasing number of accelerated electrons in the system.

Another factor contributing to the overall PL quenching is the transport of carriers away from the laser generation spot by the SAW. This effect can be evaluated by calculating the minimum value of carrier mobility $\left(\mu_{\text {carrier }}\right)$ required for an efficient acoustic transport. The SAW propagation velocity along the $\mathrm{X}$ direction of $\mathrm{Y}-128^{\circ} \mathrm{LiNbO}_{3}$ in our simulations is $v_{x}$ $=3959 \mathrm{~m} / \mathrm{s}$. This leads to a minimum carrier mobility of $\mu_{\text {carrier }}$ $=v_{x} / E_{x} \approx 20 \mathrm{~cm}^{2} /\left(\mathrm{V}\right.$ s) (for $E_{x}=20 \mathrm{kV} / \mathrm{cm}$ ), which is considerably smaller than values for exfoliated $\mathrm{MoSe}_{2}$ MLs on $\mathrm{SiO}_{2} / \mathrm{Si}$ substrates at low temperatures ${ }^{63}$ and $\mathrm{MoS}_{2}$ MLs on $\mathrm{LiNbO}_{3}$ at room temperature, ${ }^{17}$ thus indicating that the degree of PL quenching in our experiments is also affected by the carrier transport away from the laser excitation spot.

Figure $4 \mathrm{a}$ also shows that the PL emissions from the two excitonic complexes red-shift as the SAW power is increased. Figure $4 \mathrm{~d}$ displays the PL red-shift amplitude $(\Delta E)$ of the $\mathrm{MoSe}_{2} X^{0}$ (red circles) and $X^{-}$(blue circles) emissions as a function of $E_{x}$. We observe that, at $E_{x}=50 \mathrm{kV} / \mathrm{cm}, \Delta E=11.6$ $\mathrm{meV}$ for $X^{-}$and $9.2 \mathrm{meV}$ for $X^{0}$. The SAW effect on the PL emission energy can be understood by taking into account the contributions from the piezoelectric field $\left(\Delta E_{\text {Stark }}\right)$ and strain $\left(\Delta E_{\text {strain }}\right)$ to the band structure modulation of the TMD system: ${ }^{60,64}$

$$
\Delta E=\Delta E_{\text {Stark }}+\Delta E_{\text {strain }}=\frac{1}{2} \alpha_{X} E_{x}^{2}+a_{0} \frac{\delta V}{V}
$$

where $\alpha_{X}$ is the in-plane exciton polarizability, $a_{0}$ the hydrostatic band gap deformation potential, and $\delta \mathrm{V} / \mathrm{V}$ is the hydrostatic deformation.

The dashed gray line in Figure $4 \mathrm{~d}$ is the linear contribution $\Delta E_{\text {strain }}$ obtained from the $\delta V / V$ values calculated for each acoustic power (see the Supporting Information) by adopting the value $a_{0}=30 \mathrm{meV} / \%$ reported for $\mathrm{MoSe}_{2}$ MLs. ${ }^{60,65}$ The red and blue solid lines are fits with eq 2 (taking $\Delta E_{\text {strain }}$ into account) of the $X^{0}$ and $X^{-}$experimental $\Delta E$ values, respectively. They reproduce very well the experimental data, thus showing that the in-plane piezoelectric field $E_{x}$ is the major responsible for the PL red-shift in the high acoustic power regime. The out-of-plane component $\left(E_{y}\right)$ is approximately three times weaker than $E_{x}$ (see the Supporting Information). Therefore, the Stark shifts associated with $E_{y}$ are negligible compared to the ones induced by $E_{x}$ due to the very small ML thickness. ${ }^{12,13}$

From the fits, we extract $\Delta E_{\text {Stark }}$ for each excitonic emission, as shown by the cyan and orange dashed lines in Figure $4 \mathrm{~d}$. We obtain $\alpha_{\mathrm{X}^{0}}=(530 \pm 50) \times 10^{-5} \mathrm{meV} /(\mathrm{kV} / \mathrm{cm})^{2}$ and $\alpha_{\mathrm{X}^{-}}=$ $(630 \pm 70) \times 10^{-5} \mathrm{meV} /(\mathrm{kV} / \mathrm{cm})^{2}$ for the $\mathrm{MoSe}_{2} \mathrm{ML} X^{0}$ and $X^{-}$in-plane polarizabilities, respectively. These values are much larger than the value of $\alpha_{\mathrm{X}^{0}} \approx 10.4 \times 10^{-5} \mathrm{meV} /(\mathrm{kV} /$ $\mathrm{cm})^{2}$ recently reported for hexagonal boron nitride encapsulated $\mathrm{WSe}_{2}$ MLs. ${ }^{19}$ They are consistent with the lower binding energies and with expected values for in-plane polarizabilities for excitons in $\mathrm{MoSe}_{2}$ MLs on high dielectric constant environments. ${ }^{66}$ Moreover, the slightly larger trion in-plane polarizability is a signature of its lower binding energy, also in agreement with theoretical results. ${ }^{64}$ Figure $4 \mathrm{~d}$ also presents the red-shift observed for the excitonic emission band $\left(X^{0}+\right.$ $X^{-}$) of $\mathrm{MoS}_{2}$ (green dots). Due to the lower piezoelectric fields achieved on this device, we were able to probe only the low electric field limit on this sample. However, because of the broad $\mathrm{MoS}_{2}$ emission band, no red-shift could be detected for fields lower than $10.5 \mathrm{kV} / \mathrm{cm}(\Delta E=0)$. The small red-shifts observed for fields above this value indicate that the polarizabilities of $\mathrm{MoS}_{2}$ and $\mathrm{MoSe}_{2}$ are similar, as expected from theoretical calculations. ${ }^{64,66}$

\section{CONCLUSIONS}

In summary, we have employed the traveling in-plane piezoelectric field of acoustic waves to manipulate neutral and negatively charged exciton emission energies and recombination rates in $\mathrm{MoSe}_{2}$ and $\mathrm{MoS}_{2} \mathrm{MLs}$ on $\mathrm{LiNbO}_{3}$ substrates. The high dielectric constant of the substrate strongly decreases the neutral exciton binding energy. Although reduced, the binding energy is still larger than $k_{\mathrm{B}} T$, thus not preventing excitonic effects at room temperature. In this way, we found a balance that allowed us to demonstrate the applicability of SAWs as an effective noncontact technique (which preserves the $2 \mathrm{D}$ material crystal quality) to induce the Stark effect in ML semiconductor systems. Employing numerical simulations, we calculated the acoustic power 
generated by our transducer devices and were able to separate the strain and piezoelectric contributions of the acoustic fields to the band structure modulation of the ML systems. This allowed us to obtain experimental values for the large in-plane neutral and negatively charged exciton polarizabilities of nonencapsulated $\mathrm{MoSe}_{2}$ MLs placed on $\mathrm{LiNbO}_{3}$. We also presented a detailed analysis of the SAW-induced exciton dissociation process which, under strong dielectric screening, can only be described by a full interaction potential model. Our achievements demonstrate the potential of the acoustic modulation to control multiexciton dynamics in ML and fewlayer TMD systems for future applications.

\section{METHODS}

Sample Fabrication. Delay lines with FEUDTs were lithographically defined along the in-plane $\mathrm{X}$ direction of black Y-128 $\mathrm{LiNbO}_{3}$ substrates. All FEUDTs were fabricated with $N_{\mathrm{p}}=20$ finger pairs and designed for a fundamental acoustic wavelength of $\lambda_{\mathrm{SAW}}=$ $35 \mu \mathrm{m}$. The TMD MLs were obtained by micromechanical exfoliation from bulk $2 \mathrm{H}$ crystals from $\mathrm{HQ}$ graphene onto Nitto-tape. ${ }^{67}$ A second exfoliation was performed on a polydimethylsiloxane (PDMS) stamp placed on a glass slide. Bright field and fluorescence images were carried out using an Olympus BX51 fluorescence microscope at ambient conditions to identify the MLs. Using a micromanipulator, $\mathrm{MoSe}_{2}$ and $\mathrm{MoS}_{2}$ flakes were next transferred and placed close to the output port of FEUDTs with apertures of $W=212$ and $424 \mu \mathrm{m}$, respectively, onto the $\mathrm{LiNbO}_{3}$ substrates. The flakes were positioned in the middle of the SAW beam, as illustrated in Figure 1a. The devices have two lower acoustic resonances at approximately 112 and $222 \mathrm{MHz}$. In the experiments presented here, the $\mathrm{MoSe}_{2} \mathrm{ML}$ was probed with a SAW at $222 \mathrm{MHz}$ and the $\mathrm{MoS}_{2} \mathrm{ML}$ at $112 \mathrm{MHz}$. The main difference is that the higher electroacoustic power conversion of the $222 \mathrm{MHz}$ mode (and the smaller FEUDT aperture) allowed us to achieve much higher acoustic fields on the $\mathrm{MoSe}_{2} \mathrm{ML}$ (see the Supporting Information). We did not detect any influence of the SAW frequency in the main results presented here.

Finite Element Method Simulation. The SAW piezoelectric and strain fields experienced by the MLs were calculated using the finite element method (COMSOL Multiphysics 5.5, www.comsol. com). By taking into account the FEUDT design $\left(\lambda_{\mathrm{SAW}}, W\right.$, and $\left.N_{\mathrm{p}}\right)$ and the $\mathrm{LiNbO}_{3}$ substrate mechanical/piezoelectric constants, ${ }^{57,68}$ we were able to simulate the radio frequency scattering $(S)$ parameter measurements of our devices. From the nominal power applied to each FEUDT in the experiments, we calculate the amplitude of the SAW strain and piezoelectric field at the surface where each ML sits. The latter allowed us to compare the experiments performed on the $\mathrm{MoSe}_{2}$ and $\mathrm{MoS}_{2}$ MLs placed on different devices. Therefore, the results are presented as a function of $E_{x}$, which is the amplitude of the in-plane SAW piezoelectric field. For the nominal and effective acoustic powers on each experiment see the Supporting Information. In the calculations, the TMD MLs were considered to be $0.6 \mathrm{~nm}$ thick. ${ }^{45}$ The $\mathrm{MoSe}_{2}\left(\mathrm{MoS}_{2}\right) \mathrm{ML}$ was assumed to be isotropic with a Young's modulus of $224 \mathrm{GPa}(246 \mathrm{GPa}) .{ }^{69}$ The piezoelectric constants for the ML materials were adopted from density functional theory simulations, ${ }^{70}$ which have been shown to agree with experimental values. ${ }^{45}$

Acoustic Modulation Optical Spectroscopy. All optical spectroscopy measurements were performed in a coldfinger $\mathrm{He}$ cryostat at temperatures down to $5 \mathrm{~K}$. Piezoelectric positioners were used for sample scanning. Optical nonresonant excitation was carried out using CW solid-state lasers with wavelengths of $457 \mathrm{~nm}$ (for $\mathrm{MoSe}_{2}$ sample) and $532 \mathrm{~nm}$ (for $\mathrm{MoS}_{2}$ sample). The $\mu$-PL was collected using 100 (for the $\mathrm{MoSe}_{2} \mathrm{ML}$ ) and 20 (for $\mathrm{MoS}_{2} \mathrm{ML}$ ) magnification optical objectives, which produce laser spot sizes of approximately 1 and $5 \mu \mathrm{m}$ on the samples, respectively. The detection was performed with a $55 \mathrm{~cm}$ single spectrometer (with $600 \mathrm{gr} / \mathrm{mm}$ gratings) and a liquid nitrogen refrigerated CCD camera. The SAW devices were excited at the resonance frequencies via a radio frequency power generator connected to the cryostat.

\section{ASSOCIATED CONTENT}

\section{Supporting Information}

The Supporting Information is available free of charge at https://pubs.acs.org/doi/10.1021/acsnano.1c06854.

Finite element simulation of the $S$-parameter response of the FEUDT devices and their acoustic beam directionality; finite element simulation of strain and piezoelectric fields on the samples; additional data for neutral exciton and trion emissions in $\mathrm{MoSe}_{2}$ and $\mathrm{MoS}_{2}$ monolayers under different SAW powers; SAW-induced heating analysis; exciton modeling under strong dielectric screening; neutral exciton binding energy calculation; photoluminescence measurements as a function of laser excitation power (PDF)

\section{AUTHOR INFORMATION}

\section{Corresponding Author}

Odilon D. D. Couto, Jr. - Instituto de Física "Gleb

Wataghin", Universidade Estadual de Campinas, 13083-859

Campinas, Brazil; @ orcid.org/0000-0002-8416-3805;

Email: odilon@ifi.unicamp.br

\section{Authors}

Diego Scolfaro - Instituto de Física "Gleb Wataghin", Universidade Estadual de Campinas, 13083-859 Campinas, Brazil; 10 orcid.org/0000-0003-1127-4990

Matheus Finamor - Instituto de Física "Gleb Wataghin", Universidade Estadual de Campinas, 13083-859 Campinas, Brazil; $\odot$ orcid.org/0000-0001-5157-6315

Luca O. Trinchão - Instituto de Física "Gleb Wataghin", Universidade Estadual de Campinas, 13083-859 Campinas, Brazil; 이이이.org/0000-0002-9062-3388

Bárbara L. T. Rosa - Departamento de Fisica, Universidade Federal de Minas Gerais (UFMG), 30123-970 Belo Horizonte, Brazil

Andrey Chaves - Departamento de Física, Universidade Federal do Ceará, 60455-900 Fortaleza, Ceará, Brazil; Department of Physics, University of Antwerp, B-2020 Antwerp, Belgium

Paulo V. Santos - Paul-Drude-Institut für Festkörperelektronik, Leibniz-Institut im Forschungsverbund Berlin e.V., 10117 Berlin, Germany

Fernando Iikawa - Instituto de Física "Gleb Wataghin", Universidade Estadual de Campinas, 13083-859 Campinas, Brazil

Complete contact information is available at:

https://pubs.acs.org/10.1021/acsnano.1c06854

\section{Author Contributions}

D.S. and M.F. carried out the experiments. D.S. performed the data analysis. L.O.T. and O.D.D.C.Jr. performed the FEM calculations. B.L.T.R. fabricated the samples. A.C. carried out the exciton modeling. D.S. and O.D.D.C.Jr. wrote the manuscript with participation of F.I. and P.V.S. O.D.D.C.Jr. conceived and supervised the experiments. All authors commented on the manuscript and discussed the results.

\section{Notes}

The authors declare no competing financial interest. 
In the interim of our review process we became aware of similar experiments reported for acoustic transport in $\mathrm{WSe}_{2}$ MLs at room temperature. ${ }^{71}$

\section{ACKNOWLEDGMENTS}

We thank S. Rauwerdink, W. Seidel, and A. Tahraoui for the fabrication of the FEUDT transducers, M. Tanabe for the technical support during the spectroscopy measurements, and R. Fandan for providing information on the experimentally measured unidirectionality of FEUDT devices. We thank E. C. Bortolucci and the Center of Semiconductor Components and Nanotechnologies (CCSNano) from the University of Campinas as well as M. V. P. dos Santos and the multiuser laboratory (LAMULT) from the Gleb Wataghin Institute of Physics. The authors gratefully acknowledge the financial support from the São Paulo Research Foundation (FAPESP) (grants 2012/11382-9, 2018/18091-6, 2019/13654-5) and Ministry of Science, Technology, and Innovation as well as the National Council for Scientific and Technological Development (CNPq) (grants 62576/2017-0, 306107/2019-8).

\section{REFERENCES}

(1) Mak, K. F.; Shan, J. Photonics and Optoelectronics of 2D Semiconductor Transition Metal Dichalcogenides. Nat. Photonics 2016, 10, 216-226.

(2) Wang, G.; Chernikov, A.; Glazov, M. M.; Heinz, T. F.; Marie, X.; Amand, T.; Urbaszek, B. Excitons in Atomically Thin Transition Metal Dichalcogenides. Rev. Mod. Phys. 2018, 90, 021001.

(3) Xu, X.; Zao, W.; Xiao, D.; Heinz, T. F. Spin and Pseudospins in Layered Transition Metal Dichalcogenides. Nat. Phys. 2014, 10, 343350.

(4) Zhao, S.; Li, X.; Dong, B.; Wang, H.; Wang, H.; Zhang, Y.; Han, Z.; Zhang, H. Valley Manipulation in Monolayer Transition Metal Dichalcogenides and Their Hybrid Systems: Status and Challenges. Rep. Prog. Phys. 2021, 84, 026401.

(5) Aivazian, G.; Gong, Z.; Jones, A. M.; Chu, R. L.; Yan, J.; Mandrus, D. G.; Zhang, C.; Cobden, D.; Yao, W.; Xu, X. Magnetic Control of Valley Pseudospin in Monolayer $\mathrm{WSe}_{2}$. Nat. Phys. 2015, 11, 148-152.

(6) Schaibley, J. R.; Yu, H.; Clark, G.; Rivera, P.; Ross, J. S.; Seyler, K. L.; Yao, W.; Xu, X. Valleytronics in 2D Materials. Nat. Rev. Mater. 2016, 1, 16055.

(7) Schmidt, R.; Arora, A.; Plechinger, G.; Nagler, P.; del Aguila, A. G.; Ballotin, M. V.; Christianen, P. C. M.; de Vasconcellos, S. M.; Schueller, C.; Korn, T.; Bratschitsch, R. Magnetic-Field-Induced Rotation of Polarized Light Emission from Monolayer WS 2 . Phys. Rev. Lett. 2016, 117, 077402.

(8) Goryca, M.; Li, J.; Stier, A. V.; Taniguchi, T.; Watanabe, K.; Courtade, E.; Shree, S.; Robert, C.; Urbaszek, B.; Marie, X.; Crooker, S. A. Revealing Exciton Masses and Dielectric Properties of Monolayer Semiconductors with High Magnetic Fields. Nat. Commun. 2019, 10, 4172.

(9) Sie, E. J.; McIver, J. W.; Lee, Y.; Fu, L.; Kong, J.; Gedik, N. Valley-Selective Optical Stark Effect in Monolayer $\mathrm{WS}_{2}$. Nat. Mater. 2015, 14, 290-294.

(10) Kim, J.; Hong, X.; Jin, C.; Shi, S. F.; Chang, C. Y. S.; Chiu, M. H.; Li, L. J.; Wang, F. Ultrafast Generation of Pseudo-Magnetic Field for Valley Excitons in WSe $\mathrm{W}_{2}$ Monolayers. Science 2014, 346, 12051208.

(11) Ye, Z.; Sun, D.; Heinz, T. F. Optical Manipulation of Valley Pseudospin. Nat. Phys. 2017, 13, 26-29.

(12) Klein, J.; Wierzbowski; Regler, A.; Becker; Heimbach, F.; Mueller, K.; Kaniber, M.; Finley, J. J. Stark Effect Spectroscopy of Mono- and Few-Layer $\mathrm{MoS}_{2}$. Nano Lett. 2016, 16, 1554-1559.

(13) Roch, J. G.; Leisgang, N.; Froehlicher, G.; Makk, P.; Watanabe, K.; Taniguchi, T.; Schoenenberger, C.; Warburton, R. J. Quantum-
Confined Stark Effect in a $\mathrm{MoS}_{2}$ Monolayer van der Waals Heterostructure. Nano Lett. 2018, 18, 1070-1074.

(14) Chakraborty, C.; Jungwirth, N. R.; Fuchs, G. D.; Vamivakas, A. N. Electrical Manipulation of the Fine-Structure Splitting of $\mathrm{WSe}_{2}$ Quantum Emitters. Phys. Rev. B: Condens. Matter Mater. Phys. 2019, 99, 045308.

(15) Pu, J.; Matsuki, K.; Chu, L.; Kobayashi, Y.; Sasaki, S.; Miyata, Y.; Eda, G.; Takenobu, T. Exciton Polarization and Renormalization Effect for Optical Modulation in Monolayer Semiconductors. ACS Nano 2019, 13, 9218-9226.

(16) Lee, J.; Pak, S.; Lee, Y. W.; Cho, Y.; Hong, J.; Giraud, P.; Shin, H. S.; Morris, S. M.; Sohn, J. I.; Cha, S.; Kim, J. M. Monolayer Optical Memory Cells Based on Artificial Trap-Mediated Charge Storage and Release. Nat. Commun. 2015, 8, 14734.

(17) Preciado, E.; Schuelein, F. J.; Nguyen, A. E.; Barroso, D.; Isarraraz, M.; von Son, G.; Lu, I.-H.; Michailow, W.; Moeller, B.; Klee, V.; Mann, J.; Wixforth, A.; Bartels, L.; Krenner, H. J. Scalable Fabrication of a Hybrid Field-Effect and Acousto-Electric Device by Direct Growth of Monolayer $\mathrm{MoS}_{2} / \mathrm{LiNbO}_{3}$. Nat. Commun. 2015, 6, 8593.

(18) Shih, F. Y.; Wu, Y. C.; Shih, Y. S.; Shih, M. C.; Wu, T. S.; Ho, P. H.; Chen, C. W.; Chen, Y. F.; Chiu, Y. P.; Wang, W. H. EnvironmentInsensitive and Gate-Controllable Photocurrent Enabled by Bandgap Engineering of $\mathrm{MoS}_{2}$ Junctions. Sci. Rep. 2017, 7, 44768.

(19) Massicotte, M.; Vialla, F.; Schmidt, P.; Lundeberg, M. B.; Latini, S.; Haastrup, S.; Danovich, M.; Davydovskaya, D.; Watanabe, K.; Taniguchi, T.; Fal'ko, V.; Thygesen, K. S.; Pedersen, T. G.; Koppens, F. H. Dissociation of Two-Dimensional Excitons in Monolayer $\mathrm{WSe}_{2}$. Nat. Commun. 2018, 9, 1633.

(20) Pak, J.; Lee, I.; Cho, K.; Kim, J. K.; Jeong, H.; Hwang, W. T.; Ahn, G. H.; Kang, K.; Yu, W. J.; Javey, A.; Chung, S.; Lee, T. Intrinsic Optoelectronic Characteristics of $\mathrm{MoS}_{2}$ Phototransistors via a Fully Transparent van der Waals Heterostructure. ACS Nano 2019, 13, 9638-9646.

(21) He, K.; Poole, C.; Mak, K. F.; Shan, J. Experimental Demonstration of Continuous Electronic Structure Tuning via Strain in Atomically Thin $\mathrm{MoS}_{2}$. Nano Lett. 2013, 13, 2931-2936.

(22) Carrascoso, F.; Li, H.; Frisenda, R.; Castellanos-Gomez, A. Strain Engineering in Single-, Bi- and Tri-Layer $\mathrm{MoS}_{2}, \mathrm{MoSe}_{2}, \mathrm{WS}_{2}$ and $\mathrm{WSe}_{2}$. Nano Res. 2021, 14, 1698-1703.

(23) Li, H.; Contryman, A. W.; Qian, X.; Ardakani, S. M.; Gong, Y.; Wang, X.; Weisse, J. M.; Lee, C. H.; Zhao, J.; Ajayan, P. M.; Li, J.; Manoharan, H. C.; Zheng, X. Optoelectronic Crystal of Artificial Atoms in Strain-Textured Molybdenum Disulphide. Nat. Commun. 2015, 6, 7381.

(24) Blundo, E.; Felici, M.; Yildirim, T.; Pettinari, G.; Tedeschi, D.; Miriametro, A.; Liu, B.; Ma, W.; Lu, Y.; Polimeni, A. Evidence of the Direct-to-Indirect Band Gap Transition in Strained Two-Dimensional $\mathrm{WS}_{2}, \mathrm{MoS}_{2}$, and $\mathrm{WSe}_{2}$. Phys. Rev. Res. 2020, 2, 012024.

(25) Mennel, L.; Paur, M.; Mueller, T. Second Harmonic Generation in Strained Transition Metal Dichalcogenide Monolayers: $\mathrm{MoS}_{2}, \mathrm{MoSe}_{2}, \mathrm{WS}_{2}$, and $\mathrm{WSe}_{2}$. APL Photonics 2019, 4, 034404.

(26) Mennel, L.; Smejkal, V.; Linhart, L.; Burgdörfer, J.; Libisch, F.; Mueller, T. Band Nesting in Two-Dimensional Crystals: An Exceptionally Sensitive Probe of Strain. Nano Lett. 2020, 20, 42424248.

(27) Feng, J.; Qian, X.; Huang, C. W.; Li, J. Strain-Engineered Artificial Atom As a Broad-Spectrum Solar Energy Funnel. Nat. Photonics 2012, 6, 866-872.

(28) Wang, W.; Ma, X. Strain-Induced Trapping of Indirect Excitons in $\mathrm{MoSe}_{2} / \mathrm{WSe}_{2}$ Heterostructures. ACS Photonics 2020, 7, 24602467.

(29) Harats, M. G.; Kirchhof, J. N.; Qiao, M.; Greben, K.; Bolotin, K. I. Dynamics and Efficient Conversion of Excitons to Trions in Non-Uniformly Strained Monolayer $\mathrm{WS}_{2}$. Nat. Photonics 2020, 14, 324-329.

(30) Raja, A.; Chaves, A.; Yu, J.; Arefe, G.; Hill, H. M.; Rigosi, A. F.; Berkelbach, T. C.; Nagler, P.; Schueller, C.; Korn, T.; Nuckolls, C.; Hone, J.; Brus, L. E.; Heinz, T. F.; Reichman, D. R.; Chernikov, A. 
Coulomb Engineering of the Bandgap and Excitons in TwoDimensional Materials. Nat. Commun. 2017, 8, 15251.

(31) Weiss, M.; Krenner, H. J. Interfacing Quantum Emitters with Propagating Surface Acoustic Waves. J. Phys. D: Appl. Phys. 2018, 51, 373001.

(32) Delsing, P.; Cleland, A. N.; Schuetz, M. J. A.; Knörzer, J.; Giedke, G.; Cirac, J. I.; Srinivasan, K.; Wu, M.; Balram, K. C.; Bäuerle, C.; Meunier, T.; Ford, C. J. B.; Santos, P. V.; Cerda-Méndez, E.; Wang, H.; Krenner, H. J.; Nysten, E. D. S.; Weiß, M.; Nash, G. R.; Thevenard, L.; et al. The 2019 Surface Acoustic Waves Roadmap. J. Phys. D: Appl. Phys. 2019, 52, 353001.

(33) Rocke, C.; Zimmermann, S.; Wixforth, A.; Kotthaus, J. P.; Böhm, G.; Weimann, G. Acoustically Driven Storage of Light in a Quantum Well. Phys. Rev. Lett. 1997, 78, 4099-4102.

(34) Stotz, J. A. H.; Hey, R.; Santos, P. V.; Ploog, K. H. Coherent Spin Transport via Dynamic Quantum Dots. Nat. Mater. 2005, 4, 585-588.

(35) Couto, O. D. D., Jr.; Iikawa, F.; Rudolph, J.; Hey, R.; Santos, P. V. Anisotropic Spin Transport in (110) GaAs Quantum Wells. Phys. Rev. Lett. 2007, 98, 036603.

(36) Fuhrmann, D. A.; Thon, S. M.; Kim, H.; Bouwmeester, D.; Petroff, P. M.; Wixforth, A.; Krenner, H. J. Dynamic Modulation of Photonic Crystal Nanocavities Using Gigahertz Acoustic Phonons. Nat. Photonics 2011, 5, 605-609.

(37) Schülein, F. J. R.; Zallo, E.; Atkinson, P.; Schmidt, O. G.; Trotta, R.; Rastelli, A.; Wixforth, A.; Krenner, H. J. Fourier Synthesis of Radiofrequency Nanomechanical Pulses with Different Shapes. Nat. Nanotechnol. 2015, 10, 512-516.

(38) Couto, O. D. D., Jr.; Lazic, S.; Iikawa, F.; Stotz, J. A. H.; Jahn, U.; Hey, R.; Santos, P. V. Photon Anti-Bunching in Acoustically Pumped Quantum Dots. Nat. Photonics 2009, 3, 645-648.

(39) Golter, D. A.; Oo, T.; Amezcua, M.; Stewart, K. A.; Wang, H. Optomechanical Quantum Control of a Nitrogen-Vacancy Center in Diamond. Phys. Rev. Lett. 2016, 116, 143602.

(40) Janker, L.; Tong, Y.; Polavarapu, L.; Feldmann, J.; Urban, A. S.; Krenner, H. J. Real-Time Electron and Hole Transport Dynamics in Halide Perovskite Nanowires. Nano Lett. 2019, 19, 8701-8707.

(41) Rezk, A. R.; Walia, S.; Ramanathan, R.; Nili, H.; Ou, J. Z.; Bansal, V.; Friend, J. R.; Bhaskaran, M.; Yeo, L. Y.; Sriram, S. Acoustic-Excitonic Coupling for Dynamic Photoluminescence Manipulation of Quasi-2D $\mathrm{MoS}_{2}$ Nanoflakes. Adv. Opt. Mater. 2015, 3, 888-894.

(42) Huang, T.; Han, P.; Wang, X.; Ye, J.; Sun, W.; Feng, S.; Zhang, Y. Theoretical Study on Dynamic Acoustic Modulation of Free Carriers, Excitons, and Trions in 2D $\mathrm{MoS}_{2}$ Flake. J. Phys. D: Appl. Phys. 2017, 50, 114005.

(43) Rezk, A. R.; Carey, B.; Chrimes, A. F.; Lau, D. W. M.; Gibson, B. C.; Zheng, C.; Fuhrer, M. S.; Yeo, L. Y.; Kalantar-zadeh, K. Acoustically-Driven Trion and Exciton Modulation in Piezoelectric Two-Dimensional $\mathrm{MoS}_{2}$. Nano Lett. 2016, 16, 849-855.

(44) Sheng, L.; Tai, G.; Yin, Y.; Hou, C.; Wu, Z. Layer-Dependent Exciton Modulation Characteristics of $2 \mathrm{D} \mathrm{MoS}_{2}$ Driven by Acoustic Waves. Adv. Opt. Mater. 2021, 9, 2001349.

(45) Zhu, H.; Wang, Y.; Xiao, J.; Liu, M.; Xiong, S.; Wong, Z. J.; Ye, Z.; Ye, Y.; Yin, X.; Zhang, X. Observation of Piezoelectricity in FreeStanding Monolayer $\mathrm{MoS}_{2}$. Nat. Nanotechnol. 2015, 10, 151-155.

(46) Cavalcante, L. S. R.; Chaves, A.; Duppen, B. V.; Peeters, F. M.; Reichman, D. R. Electrostatics of Electron-Hole Interactions in van der Waals Heterostructures. Phys. Rev. B: Condens. Matter Mater. Phys. 2018, 97, 125427.

(47) Shin, B. G.; Han, G. H.; Yun, S. J.; Oh, H. M.; Bae, J. J.; Song, Y. J.; Park, C. Y.; Lee, Y. H. Indirect Bandgap Puddles in Monolayer $\mathrm{MoS}_{2}$ by Substrate-Induced Local Strain. Adv. Mater. 2016, 28, 93789384.

(48) Tran, M. D.; Kim, J.; Kim, H.; Doan, M. H.; Duong, D. L.; Lee, Y. H. Role of Hole Trap Sites in $\mathrm{MoS}_{2}$ of Inconsistency of Optical and Electrical Phenomena. ACS Appl. Mater. Interfaces 2018, 10, 1058010586.
(49) Novoselov, K. S.; Mishchenko, A.; Carvalho, A.; Neto, A. H. C. 2D Materials and van der Waals Heterostructures. Science 2016, 353, aac9439.

(50) Ekström, M. K.; Aref, T.; Runeson, J.; Björck, J.; Boström, I.; Delsing, P. Surface Acoustic Wave Unidirectional Transducers for Quantum Applications. Appl. Phys. Lett. 2017, 110, 073105.

(51) Tuan, D. V.; Yang, M.; Dery, H. Coulomb Interaction in Monolayer Transition-Metal Dichalcogenides. Phys. Rev. B: Condens. Matter Mater. Phys. 2018, 98, 125308.

(52) Santos, P. V.; Alsina, F.; Stotz, J. A. H.; Hey, R.; Eshlaghi, S.; Wieck, A. D. Band Mixing and Ambipolar Transport by Surface Acoustic Waves in GaAs Quantum Wells. Phys. Rev. B: Condens. Matter Mater. Phys. 2004, 69, 155318.

(53) Rocke, C.; Govorov, O.; Wixforth, A.; Böhm, G.; Weimann, G. Exciton Ionization in a Quantum Well Studied by Surface Acoustic Waves. Phys. Rev. B: Condens. Matter Mater. Phys. 1998, 57, R6850R6853.

(54) Fuhrmann, D. A.; Krenner, H. J.; Wixforth, A.; Curran, A.; Prior, K. A.; Warburton, R. J.; Ebbecke, J. Noninvasive Probing of Persistent Conductivity in High Quality ZnCdSe/ZnSe Quantum Wells Using Surface Acoustic Waves. J. Appl. Phys. 2010, 1079, 093717.

(55) Li, J. Z.; Lin, J. Y.; Jiang, H. X.; Salvador, A.; Botchkarev, A.; Morkoc, $\mathrm{H}$. Nature of Mg Impurities in GaN. Appl. Phys. Lett. 1996, 69, 1474-1476.

(56) From the Computational 2D Materials Database https:// cmrdb.fysik.dtu.dk/c2db/row/MoSe2-f61b14d398c7 (accessed 07/ 30/2021)

(57) Morgan, D. Surface Acoustic Wave Filters: with Applications to Electronic Communications and Signal Processing; Academic Press: Amsterdam, 2007.

(58) Rytova, N. S. The Screened Potential of a Point Charge in a Thin Film. Moscow Univ. Phys. Bull. 1967, 22, 18.

(59) Keldysh, L. V. Coulomb Interaction in Thin Semiconductor and Semimetal Films. JETP Lett. 1979, 29, 658.

(60) Benimetskiy, F. A.; Sharov, V. A.; Alekseev, P. A.; Kravtsov, V.; Agapev, K. B.; Sinev, I. S.; Mukhin, I. S.; Catanzaro, A.; Polozkov, R. G.; Alexeev, E. M.; Tartakovskii, A. I.; Samusev, A. K.; Skolnick, M. S.; Krizhanovskii, D. N.; Shelykh, I. A.; Iorsh, I. V. Measurement of Local Optomechanical Properties of a Direct Bandgap 2D Semiconductor. APL Mater. 2019, 7, 101126.

(61) Robert, C.; Lagarde, D.; Cadiz, F.; Wang, G.; Lassagne, B.; Amand, T.; Balocchi, A.; Renucci, P.; Tongay, S.; Urbaszek, B.; Marie, X. Exciton Radiative Lifetime in Transition Metal Dichalcogenide Monolayers. Phys. Rev. B: Condens. Matter Mater. Phys. 2016, 93, 205423.

(62) Zhang, S. K.; Santos, P. V.; Hey, R. Photoluminescence Modulation by High-Frequency Lateral Electric Fields in Quantum Wells. Appl. Phys. Lett. 2001, 78, 1559-1561.

(63) Larentis, S.; Fallahazad, B.; Tutuc, E. Field-Effect Transistors and Intrinsic Mobility in Ultra-Thin $\mathrm{MoSe}_{2}$ Layers. Appl. Phys. Lett. 2012, 101, 223104.

(64) Cavalcante, L. S. R.; da Costa, D. R.; Farias, G. A.; Reichman, D. R.; Chaves, A. Stark Shift of Excitons and Trions in TwoDimensional Materials. Phys. Rev. B: Condens. Matter Mater. Phys. 2018, 98, 245309.

(65) Lloyd, D.; Liu, X.; Christopher, J. W.; Cantley, L.; Wadehra, A.; Kim, B. L.; Goldberg, B. B.; Swan, A. K.; Bunch, J. S. Band Gap Engineering with Ultralarge Biaxial Strains in Suspended Monolayer $\mathrm{MoS}_{2}$. Nano Lett. 2016, 16, 5836-5841.

(66) Pedersen, T. G. Exciton Stark Shift and Electroabsorption in Monolayer Transition-Metal Dichalcogenides. Phys. Rev. B: Condens. Matter Mater. Phys. 2016, 94, 125424.

(67) Novoselov, K. S.; Jiang, D.; Schedin, F.; Booth, T. J.; Khotkevich, V. V.; Morozov, S. V.; Geim, A. K. Two-Dimensional Atomic Crystals. Proc. Natl. Acad. Sci. U. S. A. 2005, 102, 10451.

(68) Royer, D.; Dieulesaint, E. Elastic Waves in Solids; Springer: Heidelberg, 2000. 
(69) Iguiniz, N.; Frisenda, R.; Bratschitsch, R.; Castellanos-Gomez, A. Revisiting the Buckling Metrology Method to Determine the Young's Modulus of 2D Materials. Adv. Mater. 2019, 31, 1807150.

(70) Duerloo, K. N.; Ong, M. T.; Reed, E. J. Intrinsic Piezoelectricity in Two-Dimensional Materials. J. Phys. Chem. Lett. 2012, 3, 28712876.

(71) Datta, K.; Li, Z.; Lyu, Z.; Deotare, P. B. Piezoelectric Modulation of Excitonic Properties in Monolayer WSe $\mathrm{W}_{2}$ under Strong Dielectric Screening. ACS Nano 2021, 15, 12334-12341. 